\title{
COMMUNITY OF INQUIRY AND LEARNING IN IMMERSIVE ENVIRONMENTS
}

\author{
Ross McKerlich and Terry Anderson
}

Athabasca University

\begin{abstract}
This paper describes an exploratory, observational study using a purposive sample selection to determine if the presence indicators of the well regarded Community of Inquiry model can be a useful tool to observe and assess learning events which use a Multi User Virtual Environment (MUVE) as the mode of delivery [1]. Specific research questions addressed include whether the Community of Inquiry is recognizable in a MUVE learning environment, if new presence indicators are required if observing a MUVE learning event and finally, does the community of inquiry offer a base rubric to determine the educational effectiveness of learning events which take place in a MUVE? The results are promising; while new presence indicators add breadth to understanding the nature of learning in an immersive environment the core construct of the community of inquiry does indeed transfer to this emerging learning technology.
\end{abstract}

\section{KEYWORDS}

Community of Inquiry, Multi User Virtual Environment (MUVE), Immersive Environment, Presence, Rubric, Learning Effectiveness

\section{INTRODUCTION}

Ever since the emergence of Multi User Dungeons (MUDs) and MUDs Object Oriented (MOOs) in the late 1970's, technology developers have been working to develop ever more immersive types of environments that can be widely accessed over digital networks. With the advent of 3D technology and faster Internet connections, the progress towards achieving this goal has been significant. It is predicted that immersive environments including a variety of educational applications will be adopted on a wide scale within two to three years [2]. Immersive environments offer exciting possibilities to distance educators as they have potential to significantly reduce the subjective feelings of psychological and social distance often experienced by distance education participants. Further, immersive environments can create a sense of presence [3] and flow [4] that enhance involvement and commitment to learning activity.

Just as there are many different types and platforms for immersive environments, so there are many different names to describe this new and evolving technology. For the purposes of this paper, immersive environments will be referred to as MUVEs, Multi User Virtual Environments [5]. The terms experimental and exploratory accurately describe the current higher education experience of MUVEs. To date, little is known about the educational effectiveness of immersive environments in campus based, blended or distance modes of education. The reception by educational researchers and pundits range from uncritical acclaim to skepticism, but regardless of the reaction, it is important for educators to keep abreast of technological advances to determine and enhance pedagogical value. This exploratory paper seeks to enhance our understanding of the educational potential of MUVEs by applying the tools and principles of the well regarded community of inquiry model [1] to describe and assess teaching and learning in these immersive contexts. 


\section{A. Community of Inquiry Overview}

The community of inquiry (COI) model was developed as a framework for assessing the learning process and context in online environments in the late 1990's [1]. The COI celebrates the asynchronous nature of distance education that was prevalent at that time. The model and its component parts have been confirmed and replicated using a variety of research methodologies. Further, the COI model has been applied to educational contexts beyond computer conferencing, including face-to-face interaction [6]. However, the model has not yet been used to assess immersive environments. The purpose of this exploratory paper is to determine if the community of inquiry model is useful as a theoretical framework to describe and assess the educational effectiveness of avatar-to-avatar (A2A) teaching and to identify functional, dysfunctional and perhaps new indicators of social, cognitive and teaching presence.

The COI model is likely the most frequently cited tool used to evaluate formal distance education interaction and climate. Google Scholar lists over 150 citations for each of the 4 major papers and the book developed by the original COI authors. The seminal articles associated with this work as well links to the work of numerous researchers referencing and extending this work are available at www.communitiesofinquiry.com [7].

The COI model has its roots in Dewey's (1933) practical inquiry [8], Lipman's community of inquiry [9] and Garrison's (1991) model of critical thinking [10]. It uses this theoretical work to provide conceptual order and a practical heuristic model to assess learning in an asynchronous educational experience with extensive use of threaded text discussions. The model consists of three elements essential to an educational transaction: cognitive presence, teaching presence and social presence [11].

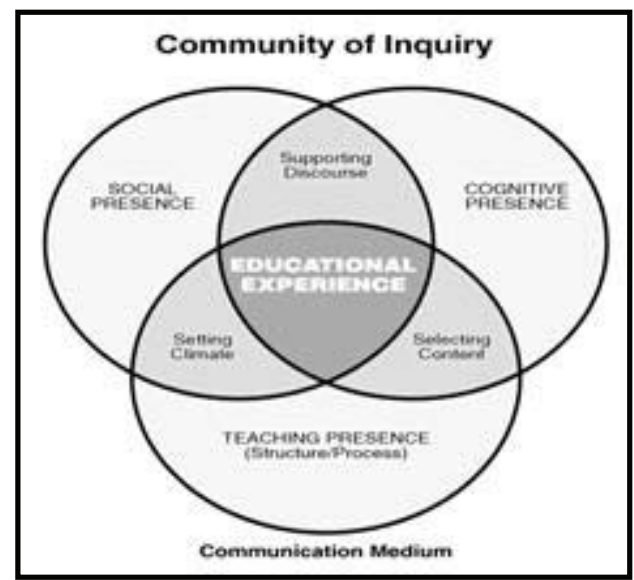

Figure 1. Community of Inquiry

Cognitive presence can be defined as the extent to which meaning can be constructed by sustained communication within a group of people [11]. Social presence is defined as the "ability to project their personal characteristics into a community thereby presenting themselves as real people to others in the group” [11]. Teaching presence involves design of the educational experience and facilitation of discourse among the group [11]. The underlying construct of the community of inquiry is that the optimal educational experience lies in the vortex of all three educational elements. It is this defining characteristic of the community of inquiry that allows it to be used as an evaluation tool. For example, if an educational experience has social presence and teaching presence but no cognitive presence then that missing element negates a critical component of the total educational experience and puts the educational effectiveness in doubt. 
As part of the original research project, Garrison et al. created indicators of all three 'presences' so that transcripts could be analyzed and the extent of the three presences could be quantified. Table 1, below, shows categories and examples of indicators of the categories:

\begin{tabular}{|lll|}
\hline Elements & Categories & Indicators (examples onIy) \\
\hline Cognitive Presence & Triggering Event & Sense of puzzlement \\
& Exploration & Information exchange \\
& Integration & Connecting ideas \\
Social Presence & Resolution & Apply new ideas \\
& Emotional Expression & Emotions \\
Teaching Presence & Open Communication & Risk-free expression \\
& Group Cohesion & Encouraging collaboration \\
& Instructional Management & Defining and initiating \\
& Building Understanding & Sharing personal meaning \\
& Direct Instruction & Focusing discussion \\
\hline
\end{tabular}

Table 1. Community of Inquiry Indicators

Indicators were identified for each of the categories with the goal that quantitative content analysis would reveal the presence and reliable indices of the extent of each presence. It is important to note that in recent years, one of the architects has acknowledged that even if the goal of the original model was to produce a quantitative tool for data analysis, qualitative approaches still yield useful results [12]. A second caveat of the Community of Inquiry model is that the indicators listed above are merely examples-there are likely different indicators for different educational environments.

\section{B. Immersive Environments Overview}

As alluded to in the introduction, the definition and defining characteristics of any new and evolving educational technology is problematic. We have followed Dede's (1995) acronym of MUVEs to describe these Multi User Virtual Environments [3]. The immersiveness of the virtual environment is a simple yet powerful focus when trying to describe this net-based context. One blogger described immersion this way: "...content is 360 degrees with height and width and depth and texture and emotions that are capable of reaching the very depths of our hearts and brains in ways no 2D media can” [13]. To explore this imagery further, using immersive environments is like jumping off a dock into a lake-once jumpers commit they are quickly and totally immersed in water. So it is with immersive environments: once we enter a multi user virtual environment we become uniquely immersed in that environment whether it is a mock-up of an existing university campus or the re-creation of a $19^{\text {th }}$ century city.

As a result of this immersion, engagement is enhanced [14], flow is supported [4], collaboration becomes both possible and supported [3] and the positive effects of new identity development and exploration are afforded [15]. In sum, these possibilities have ignited significant interest by educational researchers and teachers in exploring, testing and developing immersive contexts.

We have identified these predominant characteristics of modern internet accessible immersive environments: A MUVE is not a game, but rather a neutral and changeable environment that is co-created by the environment's designers and its users; the predominant navigation through a MUVE is by avataror a digital persona of the person using the environment; and the environment is 3 Dimensional: there is height, width and depth built using multiple media-sound, graphics, text and video. Robbins offered an extended list of characteristics of immersive environments that seems derived from Second Life, currently 
the world's largest MUVE. These characteristics include the following: that there are multiple number of users; it is an image rich environment; the environment is accessed through a Wide Area Network; the environment is persistent (that is, the environment still exists when user logs out); whatever is changed in the environment stays changed; objects in the environment are owned by their creators and access can be limited; people can create their own identities through use of avatars; there is public access to the environment; and finally, relationships with other users are collaborative and conditional-not antagonistic [16].

The avatar characteristic is unique to MUVEs and can afford an engaging and salient educational experience. Walther describes selective self-presentation as part of his hyper personal model of computer mediated communication: users in a computer mediated communication environment will exploit technology to manage impressions and "facilitate desired relationships" [17]. Walther's research was conducted in a text environment; in a 3D immersive environment learners can take advantage of rich graphic technology to present themselves in a favorable way-or perhaps not even favorable to others, but the way they want to be seen by others. Gender is chosen by the participant as well as skin color, texture- - even whether to be portrayed as a human, an animal or a new form of life. This characteristic of immersive environments adds richness and puts a new dimension on social presence: learners can project selective personal characteristics to the group often in a very creative fashion.

MUVE's allow users to gesture, smile, dance, and in many other ways express body language through their avatars' movements. Antonijevic subdivides non-verbal communication into user generated and computer generated non-verbal communication. In a recent observation study conducted in Second Life, the preliminary results show a correlation between enhanced interaction and user generated non verbal communication. Antonijevic goes on to note that "proxemic behavior observed in SL had an important communicative function, analogous to the role of proxemic cues in face-to-face interaction. Further, this type of non-verbal communication was successfully integrated with co-occurring textual discourse, enabling creation of an enriched computer mediated communication environment” [18].

Avatar use blurs the boundary between self and object. The avatar is a digital representation of a person, and the interaction, though mediated, is real: it is not the avatars' interacting but rather people interacting with people through their avatars. Lander echoes Antonijevic's comments: the real becomes shareable and interaction possibilities multiply [19]. As alluded to above, an avatar does not have to be human nor should an avatar necessarily be expected to do normal things. Wheeler and Nistor noted when describing the effects of anonymity in online culture that the more bizarre the appearance of the avatar the more bizarre the behavior [20]. This emergent behavior adds to the aforementioned salience. Dis-inhibition can increase sociability and provides new avenues for those whose actions are inhibited due to shyness or physical disability in real environments. How does this very novel form of social presence affect learning (or teaching) in immersive environments?

The actions and resulting teaching presence in MUVEs is expanded beyond classroom or other forms of online education because the teacher often is forced to act as the students' guide in this unique, changeable and often unfamiliar context. In addition, the novelty and compelling social attractiveness of MUVE's may distract learners. Arbaugh found that teachers must push students to think intellectually on the topic being taught and not be swayed or distracted by the environment itself [21].

There are a number of immersive environments available for use by educators. ActiveWorlds is used by Dede for the famed River City Project [22] and the Open Croquet (http://www.opencroquet.org) foundation offers an open source tool set for creating one's own MUVE. The MUVE that is the most 
popular today with over 5.6 million participants is Second Life [23]. Approximately 110 colleges or universities throughout the real world have a presence in Second Life [24]. These sites are used for a variety of applications - many experimental-including social gathering space, virtual representation of physical campus, institutional exposure and marketing, concert and recital space, and for teaching and learning. Academic subjects being taught include freshman composition, archeology, law, physics and research methods.

As Johnson points out, there are both advantages and disadvantages of using Second Life as an education tool yet many immersive environment educators focus only on the advantages. He notes that the main disadvantage of using Second Life for educational purposes are technological barriers: some students or educators cannot use Second life due to system requirements of the Second Life client and limited connectivity by some users-Second Life requires broadband connectivity [25]. Voice interaction has recently been added to Second Life; however it is difficult to use with large numbers and requires additional technical skills and equipment to operate effectively. Finally, and perhaps of most concern pedagogically, is the potential challenge of transferring and applying knowledge gained in MUVEs to the real world where demonstration and practice of new skills and knowledge is most productivity realized.

Although there are some purely virtual classes conducted in MUVE's many academic institutions use a blended approach: the class meets both face to face and in a MUVE. One teaching strategy in this blended approach is to use the virtual environment as an opportunity to apply knowledge that is learned in the face to face session. It is assumed that the MUVE creates a public environment that is more like a real world environment than the institutional context of formal classroom learning. For example, the blended learning approach of Harvard Law course called "CyberOne: law in the court of public opinion" allows learners to practice their argumentation skills to influence public opinion. The objective of this course is to teach prospective lawyers about the power of presenting persuasive arguments in the public domain [26]. The Harvard Law students carry out a semester long small group project in Second Life with extension school students in which, together as a group, they develop legal arguments for a moot court in Second Life (see figure 2).

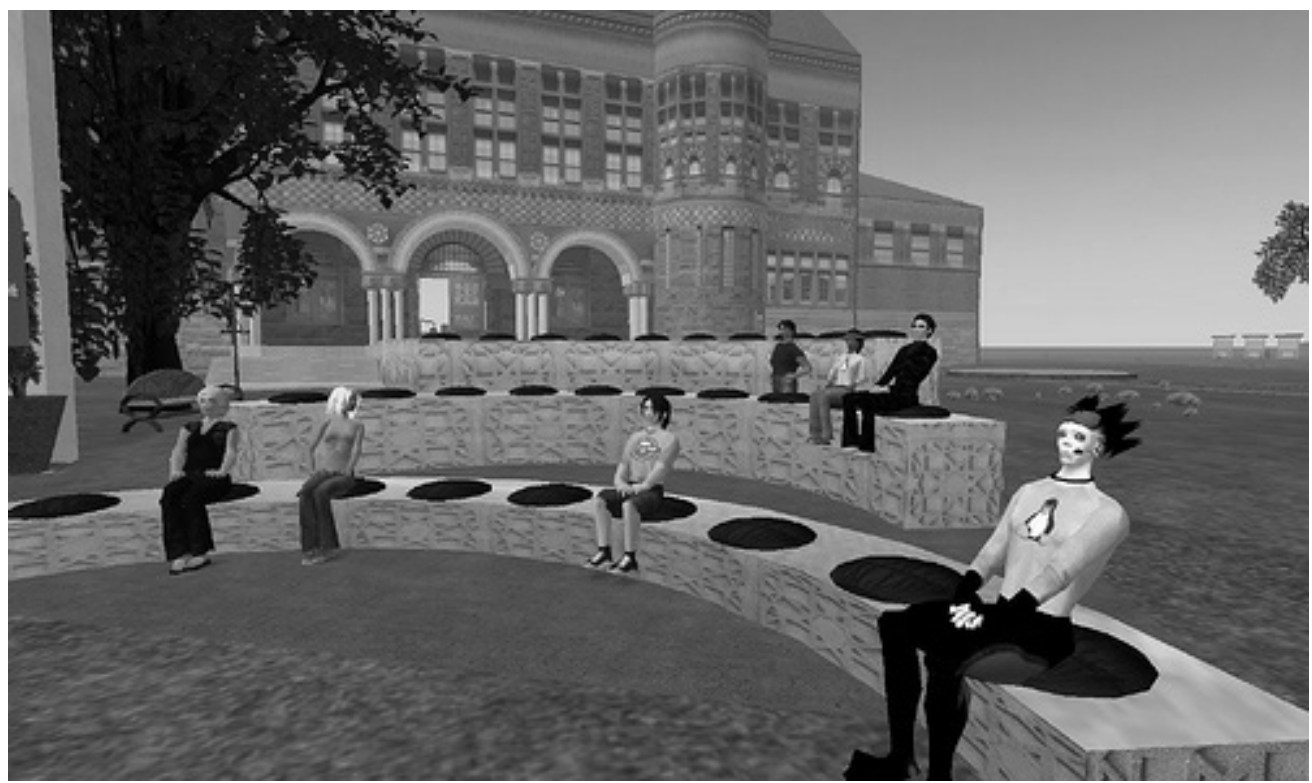

Figure 2. Harvard Law School in Second Life 


\section{METHOD}

The research that is described in this paper follows Bannan-Ritland's Integrative Learning Design framework [27]. The first stage of this research model is informed exploration-followed by enactment, local impact evaluation and broader impact evaluation [27]. Within the informed exploration stage an important research method is observation and study of the relevant educational context. We conducted an observational study using a purposive sample selection of educational events occurring in Second Life. The purposes of our observations were twofold. The first was to see if cognitive, social and teaching presences, as defined by Garrison et al's categories were recognizable in synchronous MUVE educational events. The second purpose was to determine if the indicators for each of these presences were accurate and relevant in the MUVE setting or if new or additional MUVE indicators should be identified. The overall purpose for these observations was to determine if the community of inquiry model could be used as a theoretical basis to construct an evaluation rubric for educational events in immersive environments.

The observations took place between February and April of 2007 and were conducted in the Second Life MUVE. The criteria for selecting the MUVE learning events for this study included openness to the public and a subject audience aimed at formal, post secondary level education. We also sought out diversity in learning; the size of the learning group, media and class distribution were all factors when selecting a sample MUVE learning event. The primary method of identifying potential learning events was done by monitoring the Second Life Education List--a high volume email list of interested educators. The Second Life MUVE was the study platform chosen because its client software is free to download; it currently has the largest number of active users; and there were numerous educational opportunities for study.

The six learning events were observed by a recent graduate of Athabasca University's Masters in Distance Education degree knowledgeable about the community of inquiry and its indicators. The observer's avatar during the observations was non-assuming and neutral. The observer did not overtly identify himself and was generally a passive observer. The duration for the observations was between thirty and sixty minutes. A variety of educational presentations and interaction activities were observed in the MUVE including video streaming, synchronous slide sharing, synchronous text chat with no sound, and synchronous sessions with sound. The topics also varied but all were post-secondary academic content and all events were open to the public or the instructor granted permission to the observer to attend. To note whether a category was recognizable the observer developed an observation schedule based on the community of inquiry model. These observation schedules are available on the Canadian Institute of Distance Education Research web site (http://cider.athabascau.ca).

Unlike early work by Garrison et al. we searched for evidence of broader categories of the three components of the community of inquiry, rather than detailed indicators. Garrison has noted the challenges of quantitatively analyzing text at the indicator level and questions if this level of detail is needed or enhances research validity [13]. It undoubtedly increases the challenges of reliability coding [29] when one seeks to identify all the possible indicators of complex and often projective constructs such as these three "presences." Thus, this initial work is aimed at developing more qualitative indices of presence that can be used in heuristic fashion to assess the presence of an effective community of inquiry. 


\section{RESULTS}

\section{A. Observation \#1: Immersive Environment Pedagogy Discussion}

This educational event was a workshop for educators led by an experienced MUVE educator. There were approximately ten people in attendance. The topic of the lesson was pedagogical techniques in Second Life. The location was changed at the last moment so the observer was a bit late-and this was one lesson from this observation: the teacher or his agent must ensure that the students know how to get to class. It is easy for new users to become lost and disorientated despite the navigational tools built into Second Life. Without adequate knowledge and experience, new users can be overwhelmed and continuously distracted by the rich and often disconcerting graphics, sounds and activities in this MUVE.

The observer was greeted by the instructor and answered the observer's question about how to sit down. The instructional approach was discussion group and the session was completely text based. There was a chat facilitation system - a method where a question or a comment from the class went directly to the instructor who then went through the questions which were discussed as a group-and this approach seemed to work well because it was well organized and it generated robust discussion.

We were impressed with the instructors' teaching style because it was organized and very interactive with the class. The instructor also used innovative teaching tools from his "inventory." Each avatar has an inventory that contains reusable resources and the teacher used these resources as teaching tools. An example of this occurring is when a student in the group asked what the smallest "sim" you could buy and suddenly the floor lit up with the exact size the instructor was talking about. The instructor also produced a map of the Second Life 'world' and showed the class where they were. Both resources contributed significantly to teaching presence because they demonstrated teacher's capacity to use the medium effectively, helped set the climate for learning, helped orientate study and resolved contextual confusion. The instructor used affective behaviors including use of humor, solicited student comments and expressed agreement—all indicators of social presence.

A second event that demonstrated enhanced social presence was an Instant Message session-or a private chat - with an avatar who in real life is a college instructor from the eastern United States. The observer and this avatar chatted about academic topics, and the self-exposure in instant messaging interaction created a bond of immediacy. A drawback to private instant messaging in this MUVE environment is that the multi-tasking distracted attention and thus reduced teacher presence because the observer was busy instant messaging. On the positive side, however, the dialogue resulted in increased engagement, triggering of relevant questions and exploration, all crucial components of cognitive presence.

There was also evidence of emergent and somewhat disruptive behavior in this observation: a giant squid sat down and was immediately asked to "reduce!” by the instructor, shown below in figure 3. 


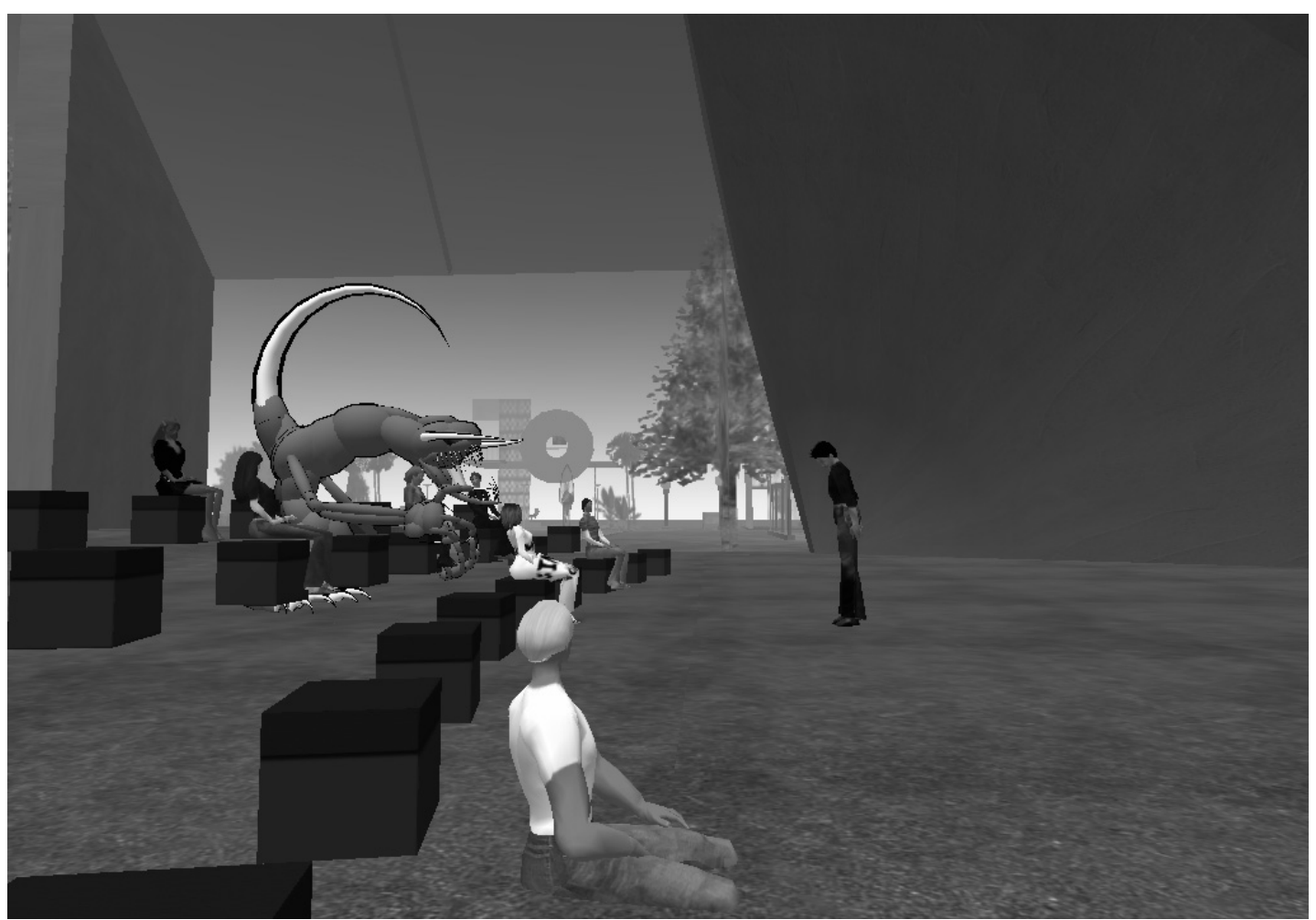

Figure 3. Emergent Behavior

The instructor's directive action was indicative again of strong teaching presence as he helped retain the climate for learning, injected knowledge of the subject domain and responded to technical concerns However, a factor which decreased teaching presence very significantly was the sudden disappearance of the instructor when his Internet connection failed. Fortunately, he reappeared in about five minutes and was able to continue with the class.

The importance of technical help underscores the need for teaching presence to include technical support. Berge noted technical support was one of three major functions of the teacher in an online environment [30]. However, in the Community of Inquiry model this technical support was subsumed as a more minor role as an indicator of direct instruction, possible because technical support was even in 1999 becoming routine or handled by technical staff and thus not the responsibility of the teacher. Obviously the novelty and complexity of MUVE's makes this assumption questionable. Secondly, use of auxiliary media (Instant Messaging, Skype, etc.) by subsets of the class was not considered in the development of the Community of Inquiry.

It seems that an additional indicator of effective teaching presence in MUVE's is control of these side channels. However, as teachers even in face-to-face contexts are finding, such control is challenging and it is unclear if side talk conversation impairs or enhances learning [31]. Finally, teacher presence seems to be enhanced by skillful simultaneous use of MUVE multimedia resources.

Based on the observation schedule, this sample MUVE learning event scored high in cognitive presence, high in teaching presence and medium in social presence. 


\section{B. Observation \#2: Guest Speaker Simulcast In a University Journalism Class}

The second observation was organized by a Canadian University professor who invited guests to attend a live session where a seasoned journalist was a guest speaker for an undergraduate journalism class. The technology was streamed audio and video. The observer used a provided Slurl (Second Life URL) that allowed users to 'teleport' directly to the auditorium where the educational event was taking place. This navigational aide was helpful to the observer and could be categorized as an additional logistics factor of teaching presence. The observer had to adjust some preferences on the Second Life client and once this was done, the observer was looking and listening at a screen with a person speaking to students in a traditional classroom at the University. In this observation, Second Life served as a distributed location increasing access to the event for those not able to attend in person.

This observation had a different feel than the chat-based observation. The observer felt detached from the event because it seemed like the speaker was concentrating on the 'real people' in front of him and the virtual participants were treated somewhat like flies on the wall. This led to decreased group cohesion which negatively impacted social presence. One method of rectifying this decreased group cohesion could have been to acknowledge and attempt interaction with the Second Life learners. The observer was greeted, however, by another avatar when he arrived in the auditorium so this increased the social presence slightly.

It was interesting to note that Second Life students all had different technical experiences based on their computer hardware. Some could hear audio, some could not. Some saw a picture upside down, some did not. These differences illustrate an important difference between analyzing community of inquiry in text based computer mediated communication and more graphic immersive environments: the technical issues in simple text based asynchronous communications are not nearly as invasive. Multi User Virtual Environments demand much higher bandwidth and computing power on the user's desk, each of which has potential to reduce access, a fundamental goal of any form of education.

This observation offered no possible additions to a MUVE based Community of Inquiry except some caveats: if the education event is a simulcast with a live speaker addressing a face-to-face audience and a MUVE audience, the speaker must acknowledge the different contexts of the participants. Non support from the simulcast instructor decreases social presence and teaching presence significantly when instruction is directed exclusively at the physically present class. A second caveat is not to assume that everyone will experience the virtual environment in the same way due to variations in technical and communication capacity. This of course complicates an evaluation of this learning context.

Based on the observations, cognitive presence was rated "medium", teaching presence was rated "low" and social presence was rated "low".

\section{Observation \#3: Guest Speaker Simulcast in a University Education Class}

The third observation of an educational event in Second Life was similar to observation \#2-a simulcast of a guest speaker. This event was sponsored by a U.S. university and included shared power point slides and audio in Second Life. The guest speaker was experienced and well respected in the Second Life educational community and obviously supportive of the educational use of MUVEs. She acknowledged the Second Life participants and had a colleague login as her avatar. This brought new meaning to teacher presence-she was in two places at once: a multimedia presentation of her real self as well as an avatar presentation of her second life avatar. The dual roles seemed to significantly reinforce teaching presence. 
The Second Life space for this observation was an open public space: there were two screens and a podium in the front. The observer logged in early because there are a maximum number of avatars allowed in a given space and as sometimes in a popular event avatars are turned away. The observer noticed later that there was an "overflow" room at another Second Life location, which again points to adding a logistics indicator for teaching presence. Based on the chat history, people seemed to know each other - the buzz was similar to pre class chatter (spontaneous interaction indicating social presence), but when the class finally got started there was not too much chat which could indicate that the Second Life students were paying attention. There was considerable international representation in this educational event-people from Slovenia, USA and someone who spoke mostly French indicating the presence of both linguistic freedom and potential language barriers.

The Second Life students could not hear sound in the beginning before class started and the observer sensed this caused some stress among Second Life attendees. Once we could hear, the sound was of good quality. The two screens were also used effectively although there was some lag in the slides.

Social presence seemed lacking-I was not greeted by anyone and no one initiated private Instant Messaging. Even though there was a teacher avatar present in Second Life any group cohesiveness between Real Life class and the Second Life class was not noticeable: we again got the impression that presenter was more focused on the physically present class. There were illustrations of affective responses during the Question \& Answer session—an “Amen” as well as salient use of emoticons by avatars.

The information was presented in a way that reflected good instructional design principles, and this contributed to teaching presence. The observer asked a question via chat, and it was responded to without delay. Other questions were also handled effectively. This also led to increased teacher presence. Finally, the presenter's slides were available to use as a resource for both real life and Second Life classes.

Logistical problems in this largest gathering of our observations were surprisingly lacking. The organizers should have done an audio test well before the class started; however, as it was, Second Life students heard the audio of the not so confident sounding technical staff which was not too reassuring and perhaps added to the stress level. There was an avatar from the host university in Second Life but he or she was not identified, so the observer could not identify this person. Other teaching presence deficiencies include the failure of the presentation to start on time, and lack of clarity about where the class was situated in Second Life.

The observer perceived that learning about the material being presented had occurred but higher levels of cognitive presence (community exploration, integration and application) in this Second Life event seemed lacking. There was no opportunity for assessment and little opportunity for the participants to engage in critical inquiry.

There was some emergent behavior-but no reaction from the Second Life class. At one point in the presentation a small avatar jumped up on to the screen displaying the PowerPoint-yet no one asked him to leave or acknowledged his presence. There were also avatars that wandered in and asked what the event was and they were answered politely and invited to stay. The avatars in this Second Life educational event were also quite normal looking - no dragon heads or tails indicating that for formal learning it may be more important to exercise one's real life in a virtual environment than to develop a second fantasy life. 
Based on the observations, cognitive presence was rated "low", teaching presence was rated "medium" and social presence was rated "low".

\section{Observation \#4: First Year English Composition Description}

The fourth observation in this exploratory study was a blended freshman composition class of small state university located in the U.S. The group met two nights a week: once in a traditional classroom and once in Second Life. This school's Second Life class meets in a gazebo like structure and the discussion was lively and well facilitated. The observer saw evidence of every category of cognitive, social and teaching presence.

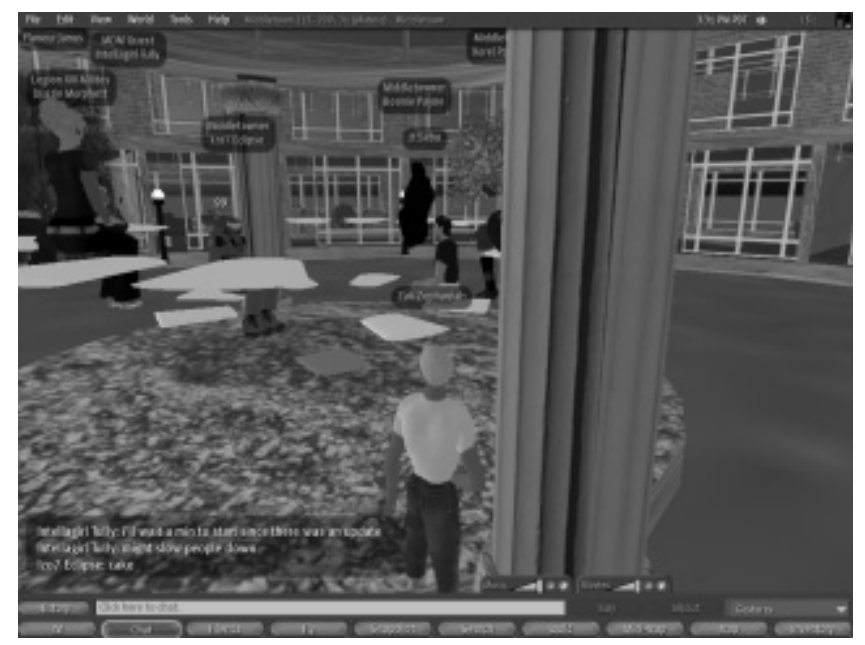

Figure 4. Freshman Comp in Second Life

The teaching style was quite Socratic in nature and the instructor asked several questions of her students to encourage critical thinking. Example questions include "What kind of sources did you find most helpful?" and "How is it hard? Where does the pressure come from?" The first part of this class was a discussion and then the group went to work on a group project. The observer checked with the instructor the next day and she noted "Last night may have seemed very chaotic but that's exactly what I intended. The students are working on a large building project and have been resistant to my efforts to make them split up and organize the work. I think that last night they finally realized that it wasn't going to work if they didn't get organized. On Tuesday night in our F2F class I fully anticipate that they'll break down and split up the tasks, finally!” (R. McKerlich, personal communication, March 30, 2007).

In terms of social presence the observer noted considerable open communication, including selfdisclosure about their real lives. One student noted "I'm back-I just put dinner on the table for my kids," and the rest of the group was interested. There was also sharing of stories about how their week had been going. There were some unusual avatars and many were welcomed with a personal greeting —an indicator of open communication - and there was considerable affectiveness, interaction and group cohesion. Immediacy was also present.

As mentioned above, there was exemplary teaching presence. The instructor directed conversation and probed deeper: "What did you learn?" She offered advice "Good to check your facts first," and also identified barriers to participation and remediation. 
Based on the observations, cognitive presence was rated "high", teaching presence was rated "high" and social presence was rated "high"

\section{E. Observation \#5: Weather Island}

The fifth observation was different from the first presentations in that it was a simulation but the observer wanted to see how an educational event of this type would fare on the observation checklist. The event was on "Weather Island," a Second Life presence for the weather channel and its objectives were to offer a learning experience for people about extreme weather. There was some teaching presence because as the observer's avatar crossed into a zone he was given audio instruction on what to do. Objectives were also stated- "to learn about extreme weather." There was also a helpful note to check preferences for a better experience which in the observer's opinion, points once more to the importance of logistics. As the observer walked towards the surf station he heard the sound of heavy surf and learned more about the locale that it represented.

There was an opportunity to experience the big waves - a free surfboard was offered — but it was not clear how to ride the surfboard. The observer did, however, experience the sound and feel of big waves so that sensation offered a new learning experience. The observer also experienced his first computer crash while using Second Life-confirming a technological barrier that seems to plague MUVEs.

The observer did not see anyone to interact with so social presence was non-existent and cognitive presence was also lacking for reasons noted above. The overall rating is not high for any of the presences-but it is a simulation that focuses exclusively on learner-content interaction and thus may have different design constraints, assessment criteria and expected outcome. One can hardly expect the development of a community of inquiry from a single learner interacting with content- though if done well significant learning may still result [32].

Based on the observations, cognitive presence was rated "low", teaching presence was rated "low" and social presence was rated "low".

\section{DISCUSSION}

The qualitative observations noted above constitute a component piece of the informed exploration stage of Bannan-Ritland's Integrative Learning Design [28]. The purpose of these observations was to determine if the community of inquiry model, developed on the basis of text based computer mediated communication, was recognizable in a MUVE learning environment. A secondary purpose was to determine if other MUVE indicators are needed to supplement the existing indicators in the community of inquiry model. The overall purpose was to determine if the Community of Inquiry can be used as a framework for evaluating educational events in immersive environments.

\section{A. Is the Community of Inquiry Recognizable in a Muve Learning Environment?}

The observer was indeed able to observe cognitive, social and teaching presence indicators in a variety of learning events that took place in an immersive environment. Based on the observation schedule and the experience of the observer it was clear that immersive environments for learning offer enhanced presence. It seems that the 'physical' presence of students and teacher configured as avatars added to the immersive 
environment educational experience.

Related to this discovery, we also learned that educational experiences, when assessed against community of inquiry indicators, offer varying degrees of overall effectiveness in terms of each educational element. For example, observation \#4, freshman composition, scored highly on social presence, teaching presence and cognitive presence and could be regarded as an example of a good educational experience in a MUVE. Observation \#2, a streamed key note lecture, scored medium on cognitive presence but low on social presence and teaching presence. As professional educators we can learn from both. The community of inquiry model thus serves well as an evaluation model for assessing MUVE educational contexts: cognitive, social and teaching presences are the standards and the categories are the criteria.

We also observed that some learning designs yield better educational results than others. This is not surprising given the importance of the learning design relative to the use of the technology itself [33]. For example, audio and slide share seem to provide a better pedagogical solution than streaming video and audio because it is easier for the learner to see the slides and the movement of the presenter and streamed audience can be distracting - especially if it is of low quality.

Finally, we learned that an observer could rarely see all community of inquiry indicators in every educational event - especially those in which learning is designed for an individual experience such as the weather simulation demonstrated. The reason for observing the simulation was to determine if the Multi User Virtual Environment Education Evaluation Tool (MUVEEET) observation checklist (see appendix) would be effective for evaluating every educational event in a MUVE. It is not-and while the MUVE simulation observation checklist is an interesting first step, considerable further systematic observation and analysis is required.

\section{B. Are New Indicators Required if the Community of Inquiry is Used as a Base Rubric for Evaluating MUVE Learning Environments?}

We found that some elements of multi-user virtual environments enhance the existing categories. For example, participants' reactions to emergent or bizarre behavior are excellent tests of the open communication category of social presence. Also, if a learning event is simulcast in two different places-one virtual and one real—-then this puts constraints on group cohesiveness if the instructor is not careful to address and involve both contexts.

The original definition of social presence from the COI model is "the ability of participants.... to project their personal characteristics into the community, thereby presenting themselves to the other participants as 'real people'” [1, p91.] Obviously, avatars are not 'real people' and many users take advantage of the creative construction tools available to create avatar images of themselves that are unlike their real selves - for example gender swapping or even adding animal or mythical extensions to their avatars. Thus, extensions of categories demonstrating social presence would include the richer capacity to express body language through avatar construction, gestures and voice. It seems likely that social presence is more profoundly affected in MUVE environments in comparison to the asynchronous text environment. However, these enhanced social presence affordances will likely lead (at least novice users) to embarrassments and breach of cultural norms, as much as they enhance learners' comfort and ease of participation in a MUVE based COI. Thus, considerable attention needs to be placed on supporting and in some cases constraining social presence if a learning environment encourages learners to act as their real selves. However, the definition of social presence is itself challenged in these contexts, as defining what is 'real' remains an ontological challenge for many users. 
We also note the enhanced importance of logistics, user competence and navigational skill needed to operate effectively in immersive environments. Do students know how to get to class? Can they hear? See? Do they have the knowledge to navigate and complete tasks related to their learning? It seems worthwhile to add efforts by teacher to reduce or remove logistic concerns as MUVE criteria of teaching presence. Another criteria addition to teaching presence is the extent to which the teacher uses the many and diverse set of tools available to him or her. For example, in the first observation the very skillful instructor used many innovative tools for the benefit of his class-including the showing of a huge map, allowing the class to visualize their location in the immersive environment. These tools or teaching/learning objects seem to add pedagogical value to instruction in immersive environments.

Cognitive presence is critical in any learning event, and some categories could be added within the context of MUVE criteria for a COI. Is the MUVE a stand-alone educational environment, or is it an addition to more traditional online tools such as learning management systems and blogs? Learning technologists are working to integrate MUVEs with other educational tools such as Sloodle (Second Life + Moodle) [33]. The integration of a learning management system with a MUVE provides another venue for the student to demonstrate critical thinking. It may not be obvious by observation if a student in Second Life comprehends the material under discussion or presentation, but this can be assessed using quizzes or postings on a course management system. A final MUVE criterion is to determine if there are unique assessment opportunities and means to demonstrate effective critical thinking. For example integration or applications of knowledge into activities or artifacts created in the MUVE are indicators of cognitive presence.

\section{Could Community of Inquiry Be Used as a Base Rubric for Evaluating Educational Events in Immersive Environments?}

Based on the data contained in the observation schedules and subsequent discussion, we believe that the community of inquiry can be used as a tool to describe and assess educational experiences and contexts in MUVEs. We conclude that the essential constructs of the community of inquiry model apply to immersive environments that expand the modes of communication to include real time text, visual and verbal cues. We further suggest that developing this kind of tool aligns with the original mandate of the Community of Inquiry: to evaluate educational experience in computer mediated contexts [1].

The observations described above reveal that an educational event in an immersive environment may have varying degrees of social, teaching and cognitive presence. We have made the first steps at developing a tool, shown in Appendix 1, which includes values for each element of the Community of Inquiry that a trained, non-biased evaluator could assign. The end result would be a MUVEEET score which could quantify the quality of an educational event in an immersive environment. This MUVEEET score could help earn a place for immersive environments in the education technology tool chest.

Drawbacks to use of the proposed tool include the requirement for synchronous use during an educational event. Real time observation is required because visual and verbal cues are used in immersive environments and part of the value of these environments is the immersion experience itself. With the use of screen recording tools this drawback could be minimized to some extent. Benefits of the proposed evaluation tool include its utility and it currency in meeting emerging needs in the educational community.

This study is a preliminary work that does not delve deeply into the methodological issues needed to validate a quantitative instrument; neither does it delve deeply into the phenomenological experience of 
learners or teachers in MUVE contexts. Nonetheless, we believe the study it is a useful first step that helps us understand learning and teaching in these emergent contexts. The following questions need to be addressed to validate the MUVE education evaluation tool:

If the categories above can be applied to immersive environments, is it possible (or even a good idea) to assign values to each indicator? Should there be weighted values or all the same?

What is the unit of analysis? - A time based function such as so many minutes of MUVE activity, or the whole event? Or a whole series of events?

How important is it for multi-coder reliability to be established, given the subjective nature of the experience of MUVE learning?

How does one establish validity for the MUVEET, especially since learners may be experiencing any of the three presences, without actually moving their avatars or interacting with the class?

Does the MUVEEET provide meaningful guidelines or assessments for both researchers and practitioners working in immersive environments?

\section{CONCLUSION}

Based on Bannan-Ritland's Integrative Learning Design Model [28] one component of the informed exploration stage has been completed by observing five diverse educational events in a Multi User Virtual Environment. Most of the key elements of the community of inquiry model were recognizable and the value of an evaluation rubric based on the COI model was noted. This proposed MUUVET instrument applies Garrison, Anderson \& Archer's Community of Inquiry model [1] to immersive environments for the purpose of evaluating educational events. The MUVEEET is based on sound academic theory and can help innovative educators justify an immersive environment educational event. The instrument needs further development, and work on its validity and reliability assessment; nonetheless it offers a first step towards evaluating this emerging educational context.

Just as innovative educators were once excited about the possibilities of text-based computer mediated communication there is great excitement about immersive environments. The Community of Inquiry model could serve both and become a standard for assessing excellence in distance education regardless of what technology is used from the educator's tool chest. 


\section{APPENDIX: MULTI USER VIRTUAL ENVIRONMENT EDUCATION EVALUATION TOOL (MUVEET)}

\begin{tabular}{|c|l|}
\hline Date & \\
\hline Event & \\
\hline Topic & \\
\hline MUVE & \\
\hline Evaluator & \\
\hline
\end{tabular}

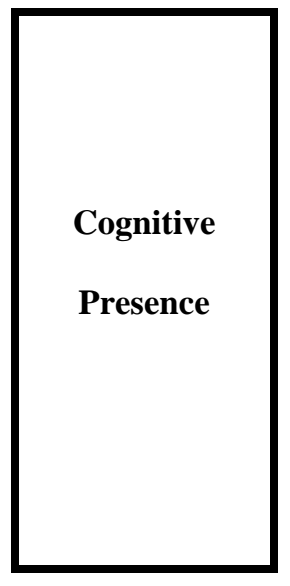

\begin{tabular}{|l|l|l|}
\hline \multicolumn{1}{|c|}{ Categories } & Observed & Example \\
\hline Triggering Event & & \\
\hline Exploration & & \\
\hline Integration & & \\
\hline Resolution & & \\
\hline
\end{tabular}

\begin{tabular}{|l|}
\hline \\
Teaching \\
Presence \\
\end{tabular}

\begin{tabular}{|l|l|l|}
\hline \multicolumn{1}{|c|}{ Muve Criteria } & Observed & Example \\
\hline Integrated Education & & \\
Tools & & \\
\hline $\begin{array}{l}\text { Use of Enhance } \\
\text { Multimedia }\end{array}$ & & \\
\hline Mediated Assessment & & \\
\hline
\end{tabular}

\begin{tabular}{|c|}
\hline \\
Social \\
Presence \\
\\
\end{tabular}

\begin{tabular}{|l|l|l|}
\hline \multicolumn{1}{|c|}{ Categories } & Observed & Example \\
\hline Design \& Organization & & \\
\hline Facilitating Discourse & & \\
\hline Direct Instruction & & \\
\hline
\end{tabular}

\begin{tabular}{|l|l|l|}
\hline \multicolumn{1}{|c|}{ Muve Criteria } & Observed & Example \\
\hline Logistical Focus & & \\
\hline Side Channel Control & & \\
\hline Teacher Representation & & \\
\hline
\end{tabular}

\begin{tabular}{|l|l|l|}
\hline \multicolumn{1}{|c|}{ Categories } & Observed & Example \\
\hline Effective Expression & & \\
\hline Open Communication & & \\
\hline Group Cohesion & & \\
\hline
\end{tabular}

\begin{tabular}{|l|l|l|}
\hline \multicolumn{1}{|c|}{ Muve Criteria } & Observed & Example \\
\hline $\begin{array}{l}\text { Do other avatars support } \\
\text { emotive expression? }\end{array}$ & & \\
\hline $\begin{array}{l}\text { Reference to Real life } \\
\text { among avatars }\end{array}$ & & \\
\hline $\begin{array}{l}\text { Initiation of after class } \\
\text { activities }\end{array}$ & & \\
\hline
\end{tabular}




\section{REFERENCE LIST}

1. Garrison, R., T. Anderson, \& W. Archer. Critical inquiry in text-based environment: Computer conferencing in higher education. The Internet and Higher Education 2(2-3): 87-105, 2000.

2. New Media Consortium. 2007 Horizon Report. 2007. http://www.nmc.org/pdf /2007_Horizon_Report.pdf. Creative Commons License.

3. Dede, C. Planning for neomillennial learning styles. Educause Quarterly 28(1): 1995. http://www .educause.edu/pub/eq/eqm05/eqm0511.asp.

4. Csikszentmihalyi, M. Flow: The Psychology of Optimal Experience. New York: Harper \& Row, 1990. http://www.debateit.net/improvethought/flow1.htm.

5. Dede, C. Multi-user virtual environments. Educause Review 38(3): 60-61, 2003.

6. Arbaugh, J. B. An empirical verification of the community of inquiry framework. Journal of Asynchronous Learning Networks 11(1): 2007. http://www.sloan-c.org/publications/jaln/v11n1/.

7. Garrison, D. R., T. Anderson, W. Archer, \& L. Rourke. Communities of Inquiry Web Site. 2007. Available at http://www.communitiesofinquiry.com/.

8. Dewey, J. How We Think. Boston: Heath, 1933.

9. Lipman, M. Thinking in Education. Cambridge: Cambridge University Press, 1991.

10. Garrison, D. R. Critical thinking in adult education: A conceptual model for developing critical thinking in adult learners. International Journal of Lifelong Education 10(4): 287-303, 1991.

11. Garrison, D. R., T. Anderson, \& W. Archer. Critical thinking and computer conferencing: A model and tool to assess cognitive presence. American Journal of Distance Education 15(1): 7-23, 2001.

12. Garrison, D. R. Online community of inquiry review: Social, cognitive and teaching presence Issues. Journal of Asynchronous Learning Networks 11(1): 2007.

13. Zimmer, L. Text 100 and PR in Second Life: A Long Way to Go. Message posted to Business Communicators of Second Life, 2006. http://freshtakes.typepad.com/sl communicators/2006/09 text 100 and pr.html.

14. Robbins, S. "Engagement in Second Life Learning”, presented at the Second Life Best Practices in Education [online], 2005. http://www.slideshare.net/intellagirl/engagement-in-second-life-learning/.

15. Turkle, S. Life on the Screen: Identity in the Age of the Internet. New York: Simon \& Schuster, 1995.

16. Robbins, S. “Ten Characteristics of Virtual Environments,” 2006. http://www.secondlife.intellagirl .com/2006/12/26/cve-muve-mmoe-mmorpg-whats-the-difference/.

17. Walther, J. B. Computer-mediated communication: Impersonal, interpersonal and hyper personal interaction. Communication Research 20(1): 3-43, 1996.

18. Antonijevic, S. "Second Life, Second Body: A Microethnographic Analysis of Nonverbal Communication in "Second Life" Virtual Environment". Paper presented at Internet Research 8.0: Let's Play, Vancouver, Canada, October, 2007. http://conferences.aoir.org /viewabstract.php?id=1120\&cf $=6$.

19. Lander, D. A. The consuming (no) body of online learners: Re-membering e-communities of practice. Studies in Continuing Education 27(2): 155-174, 2005.

20. Wheeler, S. \& N. Nistor. Human behavior in the online subculture. In N. Nistor, S. English \& S. Wheeler (Eds.), Towards the Virtual University-International On-Line Learning Perspectives, 119130. Greenwich, CT: Information Age Publishing, 2003.

21. Arbaugh, J. B. (2005b). Is there an optimal design for on-line MBA courses? Academy of Management Learning \& Education 4: 135-149, 2005.

22. Dede, C., D. J. Ketelhut, J. Clark, B. Nelson, \& C. Bowman. “Students' motivation and learning of science in a multi-user virtual environment”. Paper presented at the American Educational Research Association Conference, Montreal, Canada, 2005.

23. Second Life. http://secondlife.com/. 
24. Institutions and Organizations. Institutions and Organizations in SL [wiki] http://simteach .com/wiki/index.php?title=Institutions_and_Organizations_in_SL\#UNIVERSITIES.2C_COLLEGES _.26_SCHOOLS.

25. Johnson, N. The education potential of Second Life. Columbus, Ohio: Ohio State University, 2006.

26. Harvard University. Cyberone: Law in the court of public opinion [web site]. http://blogs.law.harvard.edu/cyberone/.

27. Bannan-Ritland, $\mathbf{B}$. The role of design in research: The integrative learning design framework. Educational Researcher 32: 21-24, 2003.

28. Rourke, L. \& T. Anderson. Validity issues in quantitative computer conference transcript analysis. Educational Technology Research and Development. In Press.

29. Berge, Z., "The role of the online instructor/facilitator" [online document]. http://star.ucc.nau.edu/ mauri/moderate/teach_online.html.

30. Anderson, T., \& D. R. Garrison. Transactional issues in distance education: The impact of design in audio teleconferencing. American Journal of Distance Education 9(2): 27-45, 1995.

31. Anderson, T. Getting the mix right: An updated and theoretical rationale for interaction. ITFORUM Paper \#63. http://it.coe.uga.edu/itforum/paper63/paper63.htm.

32. Clark, R. Media will never influence learning. Education Technology Research \& Development 42(2): 21-29, 1994.

33. Kemp, J., \& D. Livingstone. "Putting a Second Life 'Metaverse' skin on learning management systems". Paper presented at Second Life Education Workshop at SLCC, San Francisco, August, 2006. Manuscript submitted for publication.

\section{ABOUT THE AUTHOR(S)}

Ross McKerlich is a graduate of the Masters in Distance Education program at Athabasca University and owns Centerboard Strategic Learning, an e-learning consulting company. Email ross@centerboard.ca.

Terry Anderson is a professor and Canada Research Chair in Distance Education at Athabasca University - Canada’s Open University. Email terrya@athabascau.ca. 